\title{
Identification of fibrillogenic regions in human triosephosphate isomerase
}

Edson N Carcamo-Noriega, Gloria Saab-Rincon

Background. Amyloid secondary structure relies on the intermolecular assembly of polypeptide chains through main-chain interaction. According to this, all proteins have the potential to form amyloid structure, nevertheless, in nature only few proteins aggregate into toxic or functional amyloids. Structural characteristics differ greatly among amyloid proteins reported, so it has been difficult to link the fibrillogenic propensity with structural topology. However, there are ubiquitous topologies not represented in the amyloidome that could be considered as amyloid-resistant attributable to structural features, such is the case of TIM barrel topology.

Methods. This work was aimed to study the fibrillogenic propensity of human triosephosphate isomerase (HsTPI) as a model of TIM barrels. In order to do so, aggregation of HSTPI was evaluated under native-like and destabilizing conditions. Fibrillogenic regions were identified by bioinformatics approaches, protein fragmentation and peptide aggregation.

Results. We identified four fibrillogenic regions in the HsTPI corresponding to the $\beta 3, \beta 6$, $\beta 7$ y $\alpha 8$ of the TIM barrel. From these, the $\beta 3$-strand region (residues 59-66) was highly fibrillogenic. In aggregation assays, HsTPI under native-like conditions led to amorphous assemblies while under partially denaturing conditions (urea 3.2 M) formed more structured aggregates. This slightly structured aggregates exhibited residual cross- $\beta$ structure, as demonstrated by the recognition of the WO1 antibody and ATR-FTIR analysis. Discussion. Despite the fibrillogenic regions present in HsTPI, the enzyme maintained under native-favoring conditions displayed low fibrillogenic propensity. This amyloidresistance can be attributed to the three-dimensional arrangement of the protein, where $\beta$ strands, susceptible to aggregation, are protected in the core of the molecule. Destabilization of the protein structure may expose inner regions promoting $\beta$ aggregation, as well as the formation of hydrophobic disordered aggregates. Being this last pathway kinetically favored over the thermodynamically more stable fibril aggregation pathway. 
1 Title: Identification of Fibrillogenic Regions in Human Triosephosphate Isomerase.

2 Author names and affiliations: Edson Norberto Carcamo-Noriega ${ }^{1}$, Gloria Saab-Rincon ${ }^{1}$.

$3{ }^{1}$ Departamento de Ingeniería Celular y Biocatálisis, Instituto de Biotecnología, Universidad

4 Nacional Autónoma de México.

5 Corresponding author: Gloria Saab-Rincon, Departamento de Ingeniería Celular y Biocatálisis,

6 Instituto de Biotecnología, Universidad Nacional Autónoma de México, Av. Universidad No.

7 2001, Col. Chamilpa C.P. 62210, Cuernavaca, Morelos, México. Tel.: +52 (777) 329-1640;

$8 \quad$ Email: gsaab@ibt.unam.mx 


\section{1. Abstract}

10 Background. Amyloid secondary structure relies on the intermolecular assembly of polypeptide

11 chains through main-chain interaction. According to this, all proteins have the potential to form

12 amyloid structure, nevertheless, in nature only few proteins aggregate into toxic or functional amyloids. Structural characteristics differ greatly among amyloid proteins reported, so it has been difficult to link the fibrillogenic propensity with structural topology. However, there are ubiquitous topologies not represented in the amyloidome that could be considered as amyloidresistant attributable to structural features, such is the case of TIM barrel topology.

Methods. This work was aimed to study the fibrillogenic propensity of human triosephosphate isomerase (HsTPI) as a model of TIM barrels. In order to do so, aggregation of HsTPI was evaluated under native-like and destabilizing conditions. Fibrillogenic regions were identified by bioinformatics approaches, protein fragmentation and peptide aggregation.

Results. We identified four fibrillogenic regions in the HsTPI corresponding to the $\beta 3, \beta 6, \beta 7 \mathrm{y}$ $\alpha 8$ of the TIM barrel. From these, the $\beta 3$-strand region (residues 59-66) was highly fibrillogenic. In aggregation assays, HsTPI under native-like conditions led to amorphous assemblies while under partially denaturing conditions (urea 3.2 M) formed more structured aggregates. This slightly structured aggregates exhibited residual cross- $\beta$ structure, as demonstrated by the recognition of the WO1 antibody and ATR-FTIR analysis.

Discussion. Despite the fibrillogenic regions present in HsTPI, the enzyme maintained under native-favoring conditions displayed low fibrillogenic propensity. This amyloid-resistance can be attributed to the three-dimensional arrangement of the protein, where $\beta$-strands, susceptible to aggregation, are protected in the core of the molecule. Destabilization of the protein structure may expose inner regions promoting $\beta$-aggregation, as well as the formation of hydrophobic 
32 disordered aggregates. Being this last pathway kinetically favored over the thermodynamically

33 more stable fibril aggregation pathway.

34 


\section{Introduction}

The conversion of native soluble proteins into highly structured insoluble fibrillar assemblies is associated with several degenerative pathologies, such as Alzheimer's disease, Huntington's disease and Parkinson's disease, among others (Chiti \& Dobson, 2006). Currently, more than 30 different proteins are involved in amyloid processes that differ in their sequence, topology, size and function but share similar structural features in the fibrils formed. A common unit of the cross- $\beta$ spine structure is conserved in fibrillar assemblies, where $\beta$-strands stack to form $\beta$-sheets that grow perpendicular to the fiber axis (Jahn et al., 2010; Knowles, Vendruscolo \& Dobson, 2014; Westermark, 2005). Several fibrillogenesis mechanism models have been proposed based on kinetic, structural and morphological data (Zerovnik et al., 2011). These mechanisms differ from each other by: i) the number of aggregation pathways, ii) number of steps (conformational and oligomeric states of the protein) and iii) the cooperativity of amyloid assembly (Calamai, Chiti \& Dobson, 2005; Dovidchenko, Leonova \& Galzitskaya, 2014; Gillam \& MacPhee, 2013; Gosal et al., 2005; Powers \& Powers, 2008; Wu \& Shea, 2011; Zou et al., 2014).

\section{Regardless the mechanism followed, amyloid fibrils formation always relies on the} intermolecular interactions of the polypeptide main chain. Therefore, all polypeptide chains have the potential to form $\beta$-aggregates. However, not all proteins aggregate into cross- $\beta$ structures in the physiological environment (Baldwin et al., 2011). To observe protein aggregation, the native state must usually be destabilized to expose aggregation-prone regions (Knowles et al., 2014). It is clear that some sequences are more prone to form cross- $\beta$ structures than others (Jahn $\&$ Radford, 2008). Some experimental and computational studies have identified the inherent properties of the sequence, including the net charge, length, hydrophobicity and secondary 
58

59

60

61

62

structure propensities, as determinants of aggregation (Fernandez-Escamilla et al., 2004; Hills \& Brooks, 2007; Maurer-Stroh et al., 2010). Based on experimental evidence, several informatics approaches have been developed to identify aggregation-prone regions that could allow us to predict protein aggregation propensity.

These aggregation-prone regions are present in a large number of proteins and, in some cases, play a key role in the function or folding of the protein and, therefore, cannot be eliminated. However, evolution has developed protective mechanisms, such as improving solubility, steric hindrance and conformational restriction, to avoid the exposure of aggregation-prone regions (Richardson \& Richardson, 2002; Tzotzos \& Doig, 2010). It is clear that most of the protective mechanisms that evolved, are based on increasing the stability of the native state of the protein, suggesting that topologies with higher stabilities are less susceptible to amyloid aggregation (Baldwin et al., 2011). Thus, this trait can be selected during evolution, which may explain the rather limited number of folds observed in nature (Goldstein, 2008; Koehl \& Levitt, 2002).

A scaffold that has been recursively recruited during evolution is the TIM barrel $(\beta / \alpha)_{8}$ (Wierenga, 2001). So far, there are no reports of any TIM barrel forming fibrillar aggregates. Nevertheless, there is some evidence that links triosephosphate isomerase (TPI) with amyloid aggregation. In 1999, Contreras and coworkers found a segment (residues 186-218) from Escherichia coli TPI that shares $20 \%$ identity with the amyloid $\beta$-peptide (Contreras et al., 1999). When investigated, this fragment was able to assemble into amyloid-like fibrils with affinity to Congo red. In another work, Guix and coworkers found high levels of a nitrated variant of TPI (nitro-TPI) that was induced by amyloid $\beta$-peptide depositions in the brain tissues of Alzheimer's disease patients (Guix et al., 2009). Aggregation of in vitro nitrated rabbit TPI formed large $\beta$-aggregates with amyloid-like properties that were able to induce aggregation of 
81 the Tau protein. These data indicate a fibrillogenic potential in TIM barrels, moreover in human

82 triosephosphate isomerase (HsTPI) with interest in the physiopathology of Alzheimer's disease.

83 Therefore we considered imperative to evaluate the in vitro propensity of HsTPI to aggregate

84 into fibril conformation and search for fibrillogenic regions in its sequence.

85

86

87

88

89

90

91

92

93

94

95

96

97

\section{Materials and methods}

\subsection{Materials.}

All peptides were synthesized by Liquid Phase Peptide Synthesis (LPPS) without N- or Cterminal modifications by GenScript USA Inc. (Figure 3B). The WO1 antibody was generously donated by Dr. Ronald Wetzel from the Department of Structural Biology of the University of Pittsburgh. Hen egg-white lysozyme (HEWL) and all other reagents were purchased from Sigma-Aldrich Co (St. Louis, MO, USA).

\subsection{Expression and purification of HsTPI.}

The vector pET3a-HsTPI was kindly donated by Dr. Gomez Poyou (IFC-UNAM). This vector encodes the sequence of the wild-type HsTPI with a His-tag at the N-terminus followed by a TEV protease recognition sequence. The plasmid was transformed into the E. coli BL21Gold(DE3) strain. Transformed cells were grown in LB medium supplemented with ampicillin at $37^{\circ} \mathrm{C}$ until an absorbance of 0.6 at $600 \mathrm{~nm}$ was reached. Then, the expression of HsTPI was induced with IPTG at a final concentration of $0.2 \mathrm{mM}$. Incubation continued at $20{ }^{\circ} \mathrm{C}$ for $6 \mathrm{hrs}$. The cells were harvested and suspended in buffer A (20 mM sodium phosphate, $150 \mathrm{mM} \mathrm{NaCl}$, $\mathrm{pH}$ 7.4). The cell suspension was sonicated (five times for 30 seconds) and centrifuged at 15,000 $\mathrm{g}$ for 30 minutes. The supernatant was loaded into a Ni-NTA agarose column. The resin was washed with 10 column volumes of buffer A containing $50 \mathrm{mM}$ imidazole. HsTPI was then 
103 eluted with $300 \mathrm{mM}$ imidazole in buffer A. The purified HsTPI was dialyzed against buffer A in

104 order to eliminate the imidazole. The His $6 \mathrm{X}$ tag was cleaved using recombinant His-tagged TEV

105 protease at a ratio of 1:50 (w/w) protease/HsTPI at $4{ }^{\circ} \mathrm{C}$ overnight. To remove the His-tagged

106 TEV protease as well as any undigested HsTPI, the mixture was loaded into a Ni-NTA agarose

107 column and washed with buffer A. The effluent from the column containing the cleaved HsTPI

108 was recovered, precipitated with $75 \%$ ammonium sulfate and stored at $4{ }^{\circ} \mathrm{C}$. The protein

109 concentration was determined by measuring the absorbance at $280 \mathrm{~nm}$ using an extinction

110 coefficient of $\varepsilon=33460 \mathrm{M}^{-1} \mathrm{~cm}^{-1}$. The integrity of HsTPI was checked using a specific activity

111 assay.

112 3.3. Aggregation assays.

113 Aggregation assays were performed in 1.5-mL Eppendorf tubes containing $1 \mathrm{~mL}$ of $100 \mu \mathrm{M}$

114 HsTPI previously dialyzed against buffer A supplemented with $0.2 \%$ of sodium azide. Aggregation

115 was also performed under slightly denaturing conditions (3.2 $\mathrm{M}$ of urea in the same buffer). The

116 tubes containing the enzyme were incubated in a thermomixer (Eppendorf, series: 22670000) at

$11737^{\circ} \mathrm{C}$ and $600 \mathrm{rpm}$ for one week. HsTPI aggregation was followed through time by a

118 turbidimetric assay at $405 \mathrm{~nm}$ and by Thioflavin $\mathrm{T}$ (ThT) fluorescence. To do so, protein samples

$119(10 \mu \mathrm{L})$ were added to $140 \mu \mathrm{L}$ of filtered $10 \mu \mathrm{M}$ ThT in buffer A and the fluorescence emission

120 intensity at $485 \mathrm{~nm}$ was recorded using 96-well black clear bottom plates in a Tecan Safire

121 multimode microplate reader at an excitation of $440 \mathrm{~nm}$. After one week of incubation, the

122 aggregates were recovered by centrifugation at 25,000 $\mathrm{g}$ for $1 \mathrm{hr}$. The aggregation kinetics were

123 repeated three times with protein originating from different expression and purification batches. 
124

125

126

127

128

129

130

131

132

133

134

135

136

137

138

139

140

141

142

143

144

145

For peptide aggregation assays, desalted freeze-dried peptide was first dissolved. All but the $\beta 4, \beta 7$, and $\beta 8$ peptides were soluble in water. The $\beta 4$ and $\beta 7$ peptides were dissolved in $10 \%$ acetic acid while the $\beta 8$ peptide was dissolved in $100 \mathrm{mM}$ ammonium hydroxide. After dissolving, peptide solutions were diluted with phosphate buffer, supplemented with $0.02 \%$ of sodium azide, to a final concentration of $50 \mu \mathrm{M}$ and the $\mathrm{pH}$ was adjusted to 7.4. Large particles were removed by micro filtration $(0.45-\mu \mathrm{m}$ pore size $)$. A $1 \mathrm{~mL}$ sample of each peptide solution in 1.5-mL Eppendorf tubes was sealed and incubated at $37^{\circ} \mathrm{C}$ and $600 \mathrm{rpm}$ for 3 weeks. The final ThT fluorescence intensity and green-birefringence with Congo red were measured.

\subsection{Congo red birefringence.}

Congo red binding analysis was conducted by spectrophotometric assay. First, $10 \mu \mathrm{L}$ of an aggregate sample was added to $140 \mu \mathrm{L}$ of filtered $5 \mu \mathrm{M}$ Congo red in PBS. Congo red alone was used as a reference. The mixtures were incubated at room temperature for 30 minutes. Absorbance spectra from $400 \mathrm{~nm}$ to $700 \mathrm{~nm}$ were acquired on a Tecan Safire multimode microplate reader blanked with phosphate buffer. A maximum peak at $540 \mathrm{~nm}$ was indicative of red-green birefringence. A relative birefringence value was calculated using the ratio of absorbance at 540:490 nm, $b=\left(\mathrm{abs}_{540 \mathrm{~nm}} / \mathrm{abs}_{490 \mathrm{~nm}}\right)$ based on previous reports (Frid, Anisimov \& Popovic, 2007; Klunk, Jacob \& Mason, 1999).

\subsection{Dot-blot assay.}

To confirm cross- $\beta$ structure in the aggregates, a dot-blot assay against the anti-cross- $\beta$ WO1 antibody was performed. First, a $10 \mu \mathrm{L}$ sample was placed as a drop on a nitrocellulose membrane and allowed to dry. Non-specific binding sites were blocked with $5 \%(\mathrm{w} / \mathrm{v})$ bovine serum albumin for $1 \mathrm{~h}$ at room temperature. The membrane was incubated for 1 hour with the 
146 WO1 antibody at a dilution of 1:8000 in phosphate buffer containing 0.05\% (w/v) Tween 20 (T-

147 PBS). The unbound primary antibody was washed three times for 10 minutes with T-PBS. Then,

148 the membrane was incubated for $1 \mathrm{~h}$ at room temperature with the secondary antibody (alkaline

149 phosphate conjugated anti-mouse antibody; A3562-Sigma-Aldrich) using a dilution factor of

150 1:30,000 in T-PBS. The membrane was then washed 5 times with T-PBS for 10 minutes and

151 revealed using the BCIP ${ }^{\circledR} /$ NBT-Blue Liquid Substrate System for Membranes for 10 minutes.

152 The colorimetric reaction was stopped with MilliQ water.

153

154

155

156

157

\subsection{Transmission electron microscopy (TEM).}

The final aggregation products were placed on Formvar-coated 200 mesh copper grids for 1 minute. The grids were stained for 1 minute with $2 \%(\mathrm{w} / \mathrm{v})$ uranyl acetate and then washed once with MilliQ water. The images were recorded on a ZEISS transmission electron microscope model LIBRA 120 operating at $120 \mathrm{kV}$.

\subsection{Infrared spectroscopy.}

Fourier-transform infrared (FTIR) spectra of HsTPI samples were recorded using a Perkin Elmer-Spectrum Rx1 spectrometer equipped with a zinc selenide (ZnSe) Attenuated total reflection (ATR) accessory. Sample treatment and data recording was carried out as previously described (Shivu et al., 2013). A total of 256 accumulations at $1 \mathrm{~cm}^{-1}$ of resolution were performed in the range of $1800-1500 \mathrm{~cm}^{-1}$. Water-vapor spectrum was subtracted from all samples spectrum and then spectral intensities were normalized in the $1630 \mathrm{~cm}^{-1}$ peak using the Spekwin32 software. Furthermore, raw spectra in amide I region $\left(1700-1600 \mathrm{~cm}^{-1}\right)$ were analyzed by second-derivative with PeakFit 4.12 software using the Savitsky-Golay routine.

\subsection{Cross- $\beta$ region consensus prediction.}


Potential fibrillogenic regions were predicted using HsTPI sequence (UniProt ID P60174-1).

169

170

171

172

A consensus prediction was considered to be at least two sequence hits by any of the four different predictors used: FISH-AMYLOID, FOLD-AMYLOID, PASTA 2.0 and AMYLPRED 2. For all servers, the default parameters were used.

\subsection{Acid hydrolysis of HsTPI.}

The chemical cleavage reaction was carried out in 1.5-mL Eppendorf tubes. Ten $\mathrm{mg}$ of freeze dried HsTPI was dissolved in $1 \mathrm{~mL}$ of $10 \mathrm{mM} \mathrm{HCl}, 1 \mathrm{mM} \mathrm{DTT}$, pH 2, and incubated at $65^{\circ} \mathrm{C}$ for $8 \mathrm{hrs}$. After the incubation period, the reaction was cooled on ice and the hydrolysis pattern was analyzed by tricine SDS-PAGE stained with coomassie dye. The hydrolysis products were incubated at $37^{\circ} \mathrm{C}$ and 600 rpm for 7 days. The resulting aggregates were washed five times with water and then disaggregated with 7.4 M guanidinium chloride ( $\mathrm{Gdm}-\mathrm{HCl})$ by mixing overnight at room temperature..

\subsection{Mass spectroscopy analysis.}

The dissolved aggregates were desalted using a SepPack C18 cartridge and analyzed by nanoliquid chromatography and tandem mass spectrometry (nLC-MS/MS) with collision-induced dissociation (CID) on a LTQ-Orbitrap Velos (Thermo-Fisher Co., San Jose, CA) integrated with EASY-nLC II (Thermo-Fisher Co. San Jose, CA). For reverse chromatography, a 25-cm analytical column (750- $\mu \mathrm{m}$ inner diameter) packed with $\mathrm{C} 18$ resin was used in a continuous flow of $400 \mathrm{~nL} / \mathrm{min}$ in a $10-90 \%$ gradient of acetonitrile in $0.1 \%$ formic acid over $120 \mathrm{~min}$. All spectra were acquired in a data-dependent mode at a resolution of 60000 with an $\mathrm{m} / \mathrm{z}$ range of 300 to 1600 . Ions with a charge of $+2,+3$ and +4 were isolated for fragmentation using a normalized collision energy value of 35 and an activation Q value of 0.25 .

\section{$\underline{\text { 4. Results }}$}


190

191

192

193

194

195

196

197

198

199

200

201

202

203

204

205

206

207

208

209

210

211

212

\subsection{HsTPI aggregation.}

The $\beta$-aggregation propensity of HsTPI was evaluated by incubation with stirring for 7 days at $37{ }^{\circ} \mathrm{C}$ under native-like conditions $\left(\operatorname{HsTPI}_{n}\right)$. Additionally the incubation was also carried out in 3.2 $\mathrm{M}$ of urea $\left(\mathrm{HsTPI}_{\text {urea }}\right)$, a condition slightly destabilizing but still at the beginning of the unfolding transition (Mainfroid et al., 1996a; Mainfroid et al., 1996b). The kinetics of aggregation was followed by ThT fluorescence (Figure 1A) and by visible light dispersion monitored at $405 \mathrm{~nm}$ (Figure 1B). A slight increase in the ThT fluorescence is observed for $\mathrm{HsTPI}_{\mathrm{n}}$ after 40 hours. Nevertheless this low fluorescence intensity was not indicative of $\beta$ aggregation; the more drastic increment in turbidity, as indicated by the dispersion of visible light, suggests that it is more likely an interference of non-specific binding of the ThT to disordered aggregates, as displayed by other amorphous assemblies (Biancalana \& Koide, 2010; Nielsen et al., 2012; Scarafone et al., 2012). HsTPI ${ }_{\text {urea }}$, on the other hand, displayed a fast but small increase in turbidity (Figure 1B), while ThT fluorescence showed a more significant increase (Figure 1A). In this case, a short lag phase (about 5 hours) is observed with an elongation phase extended up to 7 days without reaching a plateau. According to this data, amorphous aggregation precedes $\beta$-aggregation of $\mathrm{HsTPI}_{\text {urea, }}$, which was not complete after 7 days of incubation. Longer incubation was not possible due the loss of protein by adhesion to the tube and microbial contamination despite that sodium azide was added. The TEM images of the final aggregation products of $\mathrm{HsTPI}_{\mathrm{n}}$ showed disordered aggregates with a fragmented appearance (Figure 1C). It is worth to mention that staining conditions were the same for both samples; however, the $\mathrm{HsTPI}_{\mathrm{n}}$ sample showed some spots that seem to be overstained. Image analysis shows a very entropic saturation of this micrograph compared to the one from $\mathrm{HsTPI}_{\text {urea }}$ , suggesting that the very dark spots are indeed reflecting a high concentration of protein 
213 aggregated. In the case of $\mathrm{HsTPI}_{\text {urea }}$ the aggregates displayed some elongated structures co-

214 aggregated with clusters of disordered aggregates that seem to be in an incomplete stage of the

215 fibrillogenic pathway. This observation is in good agreement with the slow rate of $\beta$-aggregation

216 as detected by ThT fluorescence.

217 ATR-FTIR was performed in order to evaluate the secondary structure of the aggregates

218 obtained after a week of incubation. The second-derivative of the IR spectrum of salted-out

219 HsTPI shown two maximal peaks around 1655 and $1633 \mathrm{~cm}^{-1}$ in the amide I region (Figure 2A).

220 These bands correspond to $\alpha$-helix and $\beta$-sheet structures, respectively. This second-derivative

221 ATR-FTIR spectrum was consistent with spectra of others $(\beta / \alpha)_{8}$ barrel protein in $\mathrm{H}_{2} \mathrm{O}$

222 (Baldassarre et al., 2011; Dong, Huang \& Caughey, 1990; Huang \& Dong, 2003; Kong \& Yu,

223 2007). After one week of incubation under native-favoring conditions, the secondary structure of

224 HsTPI was virtually unchanged suggesting native-like aggregation. In contrast, HsTPI $\mathrm{urea}_{\text {showed }}$

225 an increase of $\beta$-structure $\left(1624 \mathrm{~cm}^{-1}\right.$ band $)$ upon aggregation. Some residual non- $\beta$ secondary

226 structure was maintained around $1656 \mathrm{~cm}^{-1}$ indicating that $\beta$-aggregation was not complete.

227 Recent studies have sighted a clear tendency in the formation of new $\beta$-structure formation upon 228 aggregation despite the nature of the aggregates (Shivu et al., 2013; Wang et al., 2010). In this

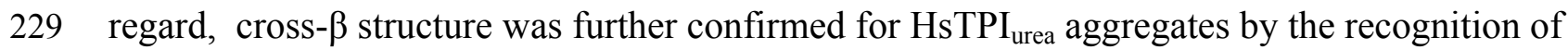

230 the anti-cross- $\beta$ WO1 antibody (O'Nuallain \& Wetzel, 2002) (Figure 2B).

\subsection{Fibrillogenic regions in HsTPI.}

In order to identify the fibrillogenic regions in HsTPI, the primary structure was submitted to four servers that use different protein aggregation prediction algorithms: FISH-AMYLOID

234 (Gasior \& Kotulska, 2014), FOLD-AMYLOID (Garbuzynskiy, Lobanov \& Galzitskaya, 2010),

235 AMYLPRED 2 (Tsolis et al., 2013) and PASTA 2.0 (Walsh et al., 2014). The prediction 
236 algorithms were based on a database of fibrillogenic sequences of prions, disease-associated 237 proteins and functional amyloid proteins (Fernandez-Escamilla et al., 2004), as well as some 238 physical-chemical principles, such as secondary structure propensity, hydrogen-bonding

239 potential, chameleonic sequences (CS), fully buried regions and structure-breaker residues such 240 as proline. The predictions reached a consensus for eight regions primarily located at the C241 terminus half of the protein (Figure 3A). The sequences of these eight regions were selected to 242 carry out aggregation assays (Figure 3B). These peptides comprise the three chameleonic 243 regions: CS-1 (residues 141-153), CS-2 (residues 168-176) and CS-3 (residues 239-249); and 244 five $\beta$-strand regions $\beta 4$ (residues 90-96), $\beta 5$ (residues 120-130), $\beta 6$ (residues 160-167), $\beta 7$ (residues 203-214) and $\beta 8$ (residues 226-236). Furthermore, the regions covering the three nonrecognized $\beta$-strand regions: $\beta 1$ (residues 6-14), $\beta 2$ (residues 37-45) and $\beta 3$ (residues 59-66), were also included for peptide aggregation assays due their inherent propensity to form parallel $\beta$-sheets and potential $\beta$-aggregation. To maximize the solubility of the synthetic peptides, extra native residues were added to the predicted regions as recommended by GenScript USA Inc (Supplemental Table S1).

During the peptide aggregation assays, the $\beta 3$ peptide became turbid by the second day of incubation followed by the $\beta 6, \beta 7$ and CS-3 peptide, where turbidity appeared by the fifth day. Measurements of the final ThT fluorescence intensities at $485 \mathrm{~nm}$ were recorded for all of the samples. The $\beta 3, \beta 6, \beta 7$ and CS-3 peptide aggregates showed a clear increase in ThT fluorescence indicating $\beta$-aggregation (Figure 4A). The $\beta 3$ aggregates showed the highest ThT fluorescence intensity, indicating a greater fibril formation for this sequence than for the $\beta 6, \beta 7$ and CS-3 sequences. In addition, aggregates of the $\beta 3, \beta 6, \beta 7$ and CS-3 peptides were tested in the spectrometric birefringence assays with Congo red (Nilsson, 2004). The four aggregates 
259 exhibited a red-green birefringence, as they displayed a maximal peak at $540 \mathrm{~nm}$ in the

260 absorbance spectrum in the range of 400-700 nm, indicative of amyloid formation, (Figure 4B).

261 The $b$ value was consistent with the ThT fluorescence measurements, indicating higher $\beta$ -

262 aggregation for $\beta 3$ and $\beta 7$ peptides. Furthermore, all peptide aggregates were examined by TEM.

263 The four peptide aggregates displayed fibrillar morphology; however, a higher degree of

264 association was achieved by the $\beta 3$ peptide since it formed a dense net of mature fibers (Figure

265 5). All others studied peptides showed amorphous aggregation or no aggregation at all.

266

267

268

269

270

271

272

273

274

275

276

277

278

279

280

281

\subsection{Acid hydrolysis of HsTPI.}

It is known that protein processing is an important factor in physiopathology of some amyloid diseases (O'Brien \& Wong, 2011; Solomon et al., 2009). This processing can be limited to specific sites in wild-type proteins, but mutations and environmental factors that destabilize the native-state can increase the fragmentation of a protein, exposing potential amyloid-prone regions. Incubation of proteins under acidic conditions gives rise to random fragmentation near Asp residues (Li et al., 2001), and favors amyloid fibril formation (Frare et al., 2004; Mishra et al., 2007). In order to investigate the potential amyloidogenic regions in HsTPI, a partial acidic hydrolysis was performed by incubating the protein at $\mathrm{pH} 2$ and $65{ }^{\circ} \mathrm{C}$ during 8 hours (Figure 6A). Assemblies of high molecular weight appeared upon partial hydrolysis, indicating a rapid association of the resulting fragments. The aggregation of fragmented HsTPI followed a nucleated polymerization mechanism with a lag phase of approximately 45 hours that reached saturation after 140 hours (Figure 6B). Fibrillar morphology was confirmed by TEM images (Figure 6C). The dissociated aggregate, after 7.4 M Gdm-HCl treatment, was analyzed by SDSPAGE and nLC-MS-MS. Figure 6D shows one main fragment below $10 \mathrm{KDa}$, a small band above $15 \mathrm{KDa}$, and other light bands at higher MW, suggesting that more than one fragment 
282 were incorporated into the amyloid fibril. However, the nLC-MS/MS analysis detected only one 283 fragment with average mass of 3143.52 Daltons (Figure 6E). We presume that the higher

284 molecular weight bands were oligomeric forms of the fragment not dissociated by Gdm-HCl.

285 The sequence of the band found by nLC-MS-MS corresponds to residues 57-85 from HsTPI, 286 covering the entire $\beta 3$-strand and most of the $\alpha 3$-helix of the $(\beta / \alpha)_{8}$ barrel.

287 As displayed in the resultant sequence, the glutamine 65 was deaminated into a glutamate 288 due to acid treatment of the protein. However, this chemical change had no effect in fibrillogenic 289 propensity since both, the synthetic ( $\beta 3$ peptide) and hydrolyzed fragment, were able to form 290 amyloid-like fibrils. It is noteworthy that despite the fact that every aspartic residue was a 291 potential cleavage site (Li et al., 2001) and therefore all predicted segments were potentially 292 covered, no other fragment was aggregated upon hydrolysis.

\section{Discussion}

\subsection{HsTPI aggregation.}

The incubation of $\mathrm{HsTPI}_{n}$ led to non-fibrillar aggregates as shown in the TEM images. ATRFTIR analysis of this aggregates revealed that $\mathrm{HsTPI}_{\mathrm{n}}$ aggregates kept most of its native secondary structure. Previous studies have remarked that globular protein can self-assemble into native-like aggregates promoted by subtle conformational changes not necessarily implying unfolding, that can be on- or off-pathway of fibrillogenesis (Bemporad \& Chiti, 2009; Jahn et al., 2006). The turbidimetric assay showed a fast increase during the first hours of incubation reaching saturation after 40 hours (Figure 1B). This change is accompanied by a small increase of ThT fluorescence (Figure 1A). The rapidity with which these aggregates were formed, as well as the low intensity of ThT fluorescence indicate rather an native-like amorphous nature of $\operatorname{HsTPI}_{n}$ aggregates. These results suggest that HsTPI under native-favoring conditions has great 
305 stability that prevents $\beta$-aggregation. In this matter and similarly to others TPIs, HsTPI thermal

306 denaturation follows a two-state irreversible model with a first-order kinetic rate constant of 7.2

$307 \times 10^{-6} \mathrm{~min}^{-1}$ at $37^{\circ} \mathrm{C}$ (Aguirre et al., 2014; Costas et al., 2009). According to this value, seven

308 days of incubation under native-favoring conditions are insufficient to allow HsTPI to visit

309 conformational states that could lead to fibrillogenesis.

310 On the other hand, the slightly destabilization of HsTPI structure with $3.2 \mathrm{M}$ urea (Mainfroid

311 et al., 1996a) showed an increase in $\beta$-aggregation according to ThT fluorescence intensities

312 (Figure 1A) suggesting that native state is protected by a high energy barrier that impedes the

313 exploration of intermediate states susceptible to $\beta$-aggregation. Higher concentrations of urea did

314 not increase $\beta$-aggregation (Supplemental Figure S2), as expected, since urea can solvate the

315 main-chain and compete for hydrogen bonds during $\beta$-aggregation (Cai et al., 2014; Hamada \&

316 Dobson, 2002; Zhang et al., 2014). In addition to ThT fluorescence, HsTPI urea aggregates were

317 analyzed by ATR-FTIR demonstrating formation of new $\beta$-structure with a characteristic lower-

318 frequency band position around $1624 \mathrm{~cm}^{-1}$ indicative of cross- $\beta$ formation (Moran \& Zanni,

319 2014; Zandomeneghi et al., 2004). Even though, recent studies have sighted a clear tendency in

320 new $\beta$-structure formation upon aggregation, despite the nature of the aggregate, the position of

321 the band below $1630 \mathrm{~nm}$ is indicative of a stronger H-bond formation as in fibrils (Shivu et al.,

322 2013; Wang et al., 2010). In this regard, cross- $\beta$ structure was further confirmed by the

323 recognition of WO1 antibody (O'Nuallain \& Wetzel, 2002).

324 It is interesting to note that destabilized HsTPI followed a nucleation-based aggregation

325 model with a short lag phase; however, the characteristic exponential fibril elongation phase of

326 the nucleated-polymerization model, was not observed. Instead, the kinetics showed a very slow

327 increase in ThT fluorescence during the seven days of incubation. A similar behavior was 
328 observed in the amyloid fibril formation of the SH3 domain of the PI3 kinase, which at pH 3.6

329 formed amorphous aggregates (1-3 h) with the posterior appearing of curly fibrils (5 days)

330 (Bader et al., 2006). It was suggested in this work that amorphous aggregates were energetically

331 more favorable than the nucleation needed for fibril formation. In contrast to HsTPI ${ }_{\text {urea }}$, the

332 aggregation of the fibrillogenic fragment found upon acid hydrolysis exhibited a longer lag phase

333 with an exponential elongation of amyloid fibrils in a clear nucleated-polymerization model

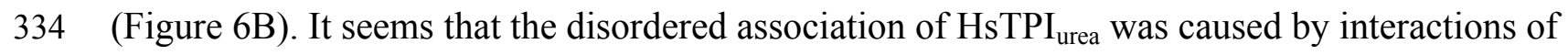

335 the rest of the protein, not present in the fragment (57-85), competing with cross- $\beta$ association,

336 delaying fibrillogenesis. Nevertheless, these interactions accelerated the intermolecular

337 association in $\mathrm{HsTPI}_{\text {urea }}$ exhibiting a shorter lag phase. It has been described a similar

338 cooperativity in both amorphous and $\beta$-sheet oligomerization, suggesting that disorder

339 aggregation could compete with $\beta$-aggregation in early steps of fibril formation (Hills \& Brooks,

340 2007; Krishnan \& Raibekas, 2009; Vetri et al., 2007). Moreover, this early amorphous

341 aggregates can play an important role in the recruitment and association of protein molecules

342 into cross- $\beta$ structure by conformational conversion (Auer et al., 2008; Johnson et al., 2012;

343 Serio et al., 2000). This proposed mechanism can explain the co-aggregation of disordered

344 structures with poor fibrillar morphology shown by $\mathrm{HsTPI}_{\text {urea }}$ aggregates, indicating that the

345 fibrillogenesis was on track but it was incomplete after 7 days of incubation.

346

\section{5.2. Fibrillogenic region of HsTPI.}

348 The identification of fibrillogenic regions was achieved through different algorithms. From

349 these, eight consensus predictions were found, from which, only 3 peptides covering the regions $\beta 6$,

$350 \quad \beta 7$ and CS-3, respectively were able to form amyloid-like aggregates as confirmed by 
351 fluorescence and birefringence assays (Figure 4). In particular, the $\beta$-aggregation of the $\beta 7$

352 peptide was consistent with previous evidence of amyloid-like aggregation in the region

353 containing the equivalent strand in the Escherichia coli TPI (Contreras et al., 1999). However,

354 Contreras et al., (1999) did not delimit the cross- $\beta$ core of the 32 residue fragment. According to

355 our results, we can infer that $\beta 7$-strand is at least one cross- $\beta$ core in the $E$. coli TPI fibrillogenic

356 fragment (186-218) due to its high identity with $\beta 7$-strand from HsTPI.

357 In addition to the consensual predicted regions, we found that the sequence comprising the

$358 \beta 3$-strand (residues 59-66), only predicted by AMYLPRED 2 server (Tsolis et al., 2013), was

359 highly fibrillogenic. All others studied peptides showed amorphous aggregation or no

360 aggregation at all. It is interesting that from an independent experiment in which fibrillogenesis

361 was investigated from acid-generated fragments of the protein, the only fragment detected from

362 dissociated fibers contains precisely the $\beta-3$ strand sequence, corroborating the great fibrillogenic

363 propensity of this region.

364

365

5.3. Amyloid protective features in HsTPI.

The presence of at least 4 cross- $\beta$ regions, including one with high fibrillogenic propensity, in

a highly expressed protein that participates in the central metabolism of any living cell, raises the question of how nature has avoided the major catastrophic events that could preclude the necessary balance to sustain life. Some structural characteristics of HsTPI topology could be consider as protective features.

371 First, all parallel $\beta$-stands are buried inside the protein, forming a $\beta$-barrel that prevents

372 further $\beta$-sheet propagation and, thereby, $\beta$-aggregation. In order to form cross- $\beta$ structure, the

373 fibrillogenic $\beta$-strands found in this study $(\beta 3, \beta 6$ and $\beta 7)$ must be exposed to the surface. 
374 However, because the amino acids constituting the $\beta$-barrel are predominantly hydrophobic, the

375 exposure of the inner core may collapse into amorphous aggregates instead of rapidly forming

376 cross- $\beta$ structures, retarding or avoiding amyloid-like aggregation.

377 In the case of solvent-exposed regions, which are more accessible for intermolecular

378 associations, there is a structural restriction avoiding the $\beta$-conformation that promotes the $\alpha$ -

379 helix or random coil conformations. This mechanism could prevent the cross- $\beta$ association of

380 CS-3 in the native fold because this region is restricted to the last $\alpha$-helix of HsTPI.

381 It is noteworthy that most of the predicted and demonstrated fibrillogenic regions are in the C-

382 terminus half of the protein. Recent studies of in vivo folding indicate that folding starts as soon

383 as the polypeptide chain leaves the ribosome tunnel (O'Brien et al., 2010). So that in vivo, by the

384 time the C-regions are exposed, the N-terminus half has probably started its folding process,

385 directing the folding of the remaining of the polypeptide, and avoiding any off-pathway

386 intermolecular interaction. Once the native state is reached its high energy barrier protects it

387 from partial unfolding that could expose these regions (O'Brien et al., 2011; Ugrinov \& Clark, 388 2010).

389 In addition to the tertiary structure of $(\beta / \alpha)_{8}$, TPI is always found as an oligomer, and more

390 frequently as a dimer. This intermolecular association contributes to an 8-fold increase in the

391 stability of the human enzyme (Mainfroid et al., 1996a). The interface region is precisely formed

392 by the loop following the $\beta 3$-strand that interdigitates into the active site of the other subunit.

393 This interaction gives extra protection to the region around the $\beta 3$-strand. Although most of the

394 efforts to perturb the dimeric interface of the protein have yielded inactive proteins (Borchert et

395 al., 1994; Borchert et al., 1995; Schliebs et al., 1997), the generation of a sufficiently active

396 monomeric variant (Saab-Rincon et al., 2001) rules out the possibility that activity is the only 
397 major selective pressure for this protein to maintain its oligomeric state. Instead, it is possible 398 that the changes in tertiary contacts in the fibrillogenic regions upon dimerization, increases the

399 energetic barrier for the formation of amyloid fibrils, as suggested by Buell et al. (Buell et al., 400 2012), which could be another selective factor to maintain TPIs as dimers.

401 Finally, it has been observed that the size of polypeptide chain could influence in the aggregation 402 of proteins (Baldwin et al., 2011; Ramshini et al., 2011; Solomon et al., 2009). The length of the 403 polypeptide chain could be associated with the number of possible conformation states in the 404 intermolecular protein association increasing the number and complexity of the aggregation 405 pathways. Since HsTPI is a medium-size protein with a compact, stable and evolution-selected 406 topology is reasonably to speculate the existence of competitive aggregation pathways, once the

407 native state is altered, that avoid cross- $\beta$ formation.

\section{$\underline{\text { 6. Conclusion }}$}

It is clear that albeit containing at least four potential amyloidogenic regions, the nature of HsTPI

410 confers protection against the formation of toxic amyloid aggregates. However, mutations or

411 post-translational modifications might affect its solubility, stability and/or folding, allowing it to

412 develop a role in amyloid diseases.

\section{7. Acknowledgments}

414 We thank Dr. Ronald Wetzel and Ravindra Kodali for kindly donating the WO1 antibody, Dr.

415 Armando Gómez Poyou† for providing the pET3a-HsTPI plasmid, Biol. Filiberto Sanchez

416 López for technical support, and the following facilities of the Instituto de Biotecnología,

417 Universidad Nacional Autónoma de México for their services at different stages of this work:

418 Laboratorio Nacional de Microscopía Avanzada, specifically, Dr. Guadalupe Zavala; 
419 Laboratorio Universitario de Proteómica, specifically Dr. Cesar Ferreira Batista; and Unidad de

420 Cómputo and Unidad de Biblioteca.

421

422

423

424

425

426

427

428

429

430

431

432

433

434

435

436

437

438

439

440

441

442

443

444

445

446

447

448

449

450

451

452

453

454

455

456

457

458

459

460

461

462

463

\section{References}

Aguirre Y, Cabrera N, Aguirre B, Perez-Montfort R, Hernandez-Santoyo A, Reyes-Vivas H, EnriquezFlores S, de Gomez-Puyou MT, Gomez-Puyou A, Sanchez-Ruiz JM, Costas M. 2014. Different contribution of conserved amino acids to the global properties of triosephosphate isomerases. Proteins 82:323-335. 10.1002/prot.24398

Auer S, Meersman F, Dobson CM, Vendruscolo M. 2008. A generic mechanism of emergence of amyloid protofilaments from disordered oligomeric aggregates. PLoS Comput Biol 4:e1000222. 10.1371/journal.pcbi.1000222

Bader R, Bamford R, Zurdo J, Luisi BF, Dobson CM. 2006. Probing the mechanism of amyloidogenesis through a tandem repeat of the PI3-SH3 domain suggests a generic model for protein aggregation and fibril formation. J Mol Biol 356:189-208. 10.1016/j.jmb.2005.11.034

Baldassarre M, Scire A, Fiume I, Tanfani F. 2011. Insights into the structural properties of D-serine dehydratase from Saccharomyces cerevisiae: an FT-IR spectroscopic and in silico approach. Biochimie 93:542-548. 10.1016/j.biochi.2010.11.009

Baldwin AJ, Knowles TP, Tartaglia GG, Fitzpatrick AW, Devlin GL, Shammas SL, Waudby CA, Mossuto MF, Meehan S, Gras SL, Christodoulou J, Anthony-Cahill SJ, Barker PD, Vendruscolo M, Dobson CM. 2011. Metastability of native proteins and the phenomenon of amyloid formation. J Am Chem Soc 133:14160-14163. 10.1021/ja2017703

Bemporad F, Chiti F. 2009. "Native-like aggregation" of the acylphosphatase from Sulfolobus solfataricus and its biological implications. FEBS Letters 583:2630-2638. http://dx.doi.org/10.1016/j.febslet.2009.07.013

Biancalana M, Koide S. 2010. Molecular mechanism of Thioflavin-T binding to amyloid fibrils. Biochim Biophys Acta 1804:1405-1412. 10.1016/j.bbapap.2010.04.001

Borchert TV, Abagyan R, Jaenicke R, Wierenga RK. 1994. Design, creation, and characterization of a stable, monomeric triosephosphate isomerase. Proc Natl Acad Sci U S A 91:1515-1518.

Borchert TV, Zeelen JP, Schliebs W, Callens M, Minke W, Jaenicke R, Wierenga RK. 1995. An interface point-mutation variant of triosephosphate isomerase is compactly folded and monomeric at low protein concentrations. Febs Lett 367:315-318.

Buell AK, Dhulesia A, White DA, Knowles TP, Dobson CM, Welland ME. 2012. Detailed analysis of the energy barriers for amyloid fibril growth. Angew Chem Int Ed Engl 51:5247-5251.

Cai Z, Li J, Yin C, Yang Z, Wu J, Zhou R. 2014. Effect of urea concentration on aggregation of amyloidogenic hexapeptides (NFGAIL). J Phys Chem B 118:48-57. 10.1021/jp407776e

Calamai M, Chiti F, Dobson CM. 2005. Amyloid fibril formation can proceed from different conformations of a partially unfolded protein. Biophys $J$ 89:4201-4210. 10.1529/biophysj.105.068726

Contreras CF, Canales MA, Alvarez A, De Ferrari GV, Inestrosa NC. 1999. Molecular modeling of the amyloid-beta-peptide using the homology to a fragment of triosephosphate isomerase that forms amyloid in vitro. Protein Eng 12:959-966.

Costas M, Rodriguez-Larrea D, De Maria L, Borchert TV, Gomez-Puyou A, Sanchez-Ruiz JM. 2009. Between-species variation in the kinetic stability of TIM proteins linked to solvation-barrier free energies. J Mol Biol 385:924-937. 10.1016/j.jmb.2008.10.056

Chiti F, Dobson CM. 2006. Protein misfolding, functional amyloid, and human disease. Annu Rev Biochem 75:333-366. 10.1146/annurev.biochem.75.101304.123901 
464

465

466

467

468

469

470

471

472

473

474

475

476

477

478

479

480

481

482

483

484

485

486

487

488

489

490

491

492

493

494

495

496

497

498

499

500

501

502

503

504

505

506

507

508

509

510

511

512

513

Dong A, Huang P, Caughey WS. 1990. Protein secondary structures in water from second-derivative amide I infrared spectra. Biochemistry 29:3303-3308.

Dovidchenko NV, Leonova EI, Galzitskaya OV. 2014. Mechanisms of amyloid fibril formation. Biochemistry (Mosc) 79:1515-1527. 10.1134/S0006297914130057

Fernandez-Escamilla AM, Rousseau F, Schymkowitz J, Serrano L. 2004. Prediction of sequencedependent and mutational effects on the aggregation of peptides and proteins. Nat Biotechnol 22:1302-1306. 10.1038/nbt1012

Frare E, Polverino De Laureto P, Zurdo J, Dobson CM, Fontana A. 2004. A highly amyloidogenic region of hen lysozyme. J Mol Biol 340:1153-1165. 10.1016/j.jmb.2004.05.056

Frid P, Anisimov SV, Popovic N. 2007. Congo red and protein aggregation in neurodegenerative diseases. Brain Res Rev 53:135-160. 10.1016/j.brainresrev.2006.08.001

Garbuzynskiy SO, Lobanov MY, Galzitskaya OV. 2010. FoldAmyloid: a method of prediction of amyloidogenic regions from protein sequence. Bioinformatics 26:326-332. 10.1093/bioinformatics/btp691

Gasior P, Kotulska M. 2014. FISH Amyloid - a new method for finding amyloidogenic segments in proteins based on site specific co-occurrence of aminoacids. BMC Bioinformatics 15:54. 10.1186/1471-2105-15-54

Gillam JE, MacPhee CE. 2013. Modelling amyloid fibril formation kinetics: mechanisms of nucleation and growth. $J$ Phys Condens Matter 25:373101. 10.1088/0953-8984/25/37/373101

Goldstein RA. 2008. The structure of protein evolution and the evolution of protein structure. Current Opinion in Structural Biology 18:170-177. http://dx.doi.org/10.1016/j.sbi.2008.01.006

Gosal WS, Morten IJ, Hewitt EW, Smith DA, Thomson NH, Radford SE. 2005. Competing pathways determine fibril morphology in the self-assembly of beta2-microglobulin into amyloid. $J$ Mol Biol 351:850-864. 10.1016/j.jmb.2005.06.040

Guix FX, Ill-Raga G, Bravo R, Nakaya T, de Fabritiis G, Coma M, Miscione GP, Villa-Freixa J, Suzuki T, Fernandez-Busquets X, Valverde MA, de Strooper B, Munoz FJ. 2009. Amyloid-dependent triosephosphate isomerase nitrotyrosination induces glycation and tau fibrillation. Brain 132:1335-1345.

Hamada D, Dobson CM. 2002. A kinetic study of beta-lactoglobulin amyloid fibril formation promoted by urea. Protein Sci 11:2417-2426. 10.1110/ps.0217702

Hills RD, Jr., Brooks CL, 3rd. 2007. Hydrophobic cooperativity as a mechanism for amyloid nucleation. $J$ Mol Biol 368:894-901. 10.1016/j.jmb.2007.02.043

Huang P, Dong A. 2003. Thermal, chemical and chemothermal denaturation of yeast enolase. Spectroscopy 17. 10.1155/2003/941801

Jahn TR, Makin OS, Morris KL, Marshall KE, Tian P, Sikorski P, Serpell LC. 2010. The common architecture of cross-beta amyloid. J Mol Biol 395:717-727. 10.1016/j.jmb.2009.09.039

Jahn TR, Parker MJ, Homans SW, Radford SE. 2006. Amyloid formation under physiological conditions proceeds via a native-like folding intermediate. Nat Struct Mol Biol 13:195-201. $10.1038 / \mathrm{nsmb} 1058$

Jahn TR, Radford SE. 2008. Folding versus aggregation: polypeptide conformations on competing pathways. Arch Biochem Biophys 469:100-117. 10.1016/j.abb.2007.05.015

Johnson SM, Connelly S, Fearns C, Powers ET, Kelly JW. 2012. The transthyretin amyloidoses: from delineating the molecular mechanism of aggregation linked to pathology to a regulatory-agencyapproved drug. J Mol Biol 421:185-203. 10.1016/j.jmb.2011.12.060

Klunk WE, Jacob RF, Mason RP. 1999. Quantifying amyloid beta-peptide (Abeta) aggregation using the Congo red-Abeta (CR-abeta) spectrophotometric assay. Anal Biochem 266:66-76. S00032697(98)92933-5 [pii]

10.1006/abio.1998.2933

Knowles TP, Vendruscolo M, Dobson CM. 2014. The amyloid state and its association with protein misfolding diseases. Nat Rev Mol Cell Biol 15:384-396. 10.1038/nrm3810 
514

515

516

517

518

519

520

521

522

523

524

525

526

527

528

529

530

531

532

533

534

535

536

537

538

539

540

541

542

543

544

545

546

547

548

549

550

551

552

553

554

555

556

557

558

559

560

561

562

563

Koehl P, Levitt M. 2002. Protein topology and stability define the space of allowed sequences. Proc Natl Acad Sci U S A 99:1280-1285. 10.1073/pnas.032405199

Kong J, Yu S. 2007. Fourier transform infrared spectroscopic analysis of protein secondary structures. Acta Biochim Biophys Sin (Shanghai) 39:549-559.

Krishnan S, Raibekas AA. 2009. Multistep aggregation pathway of human interleukin-1 receptor antagonist: kinetic, structural, and morphological characterization. Biophys $J$ 96:199-208. 10.1016/j.bpj.2008.10.002

Li A, Sowder RC, Henderson LE, Moore SP, Garfinkel DJ, Fisher RJ. 2001. Chemical cleavage at aspartyl residues for protein identification. Anal Chem 73:5395-5402.

Mainfroid V, Mande SC, Hol WG, Martial JA, Goraj K. 1996a. Stabilization of human triosephosphate isomerase by improvement of the stability of individual alpha-helices in dimeric as well as monomeric forms of the protein. Biochemistry 35:4110-4117. 10.1021/bi952692n

Mainfroid V, Terpstra P, Beauregard M, Frere JM, Mande SC, Hol WG, Martial JA, Goraj K. 1996b. Three hTIM mutants that provide new insights on why TIM is a dimer. $J$ Mol Biol 257:441-456. 10.1006/jmbi.1996.0174

Maurer-Stroh S, Debulpaep M, Kuemmerer N, Lopez de la Paz M, Martins IC, Reumers J, Morris KL, Copland A, Serpell L, Serrano L, Schymkowitz JW, Rousseau F. 2010. Exploring the sequence determinants of amyloid structure using position-specific scoring matrices. Nat Methods 7:237242. 10.1038/nmeth.1432

Mishra R, Sorgjerd K, Nystrom S, Nordigarden A, Yu YC, Hammarstrom P. 2007. Lysozyme amyloidogenesis is accelerated by specific nicking and fragmentation but decelerated by intact protein binding and conversion. J Mol Biol 366:1029-1044. 10.1016/j.jmb.2006.11.084

Moran SD, Zanni MT. 2014. How to Get Insight into Amyloid Structure and Formation from Infrared Spectroscopy. The Journal of Physical Chemistry Letters 5:1984-1993. 10.1021/jz500794d

Nielsen SB, Yde P, Giehm L, Sundbye S, Christiansen G, Mathiesen J, Jensen MH, Jensen PH, Otzen DE. 2012. Multiple roles of heparin in the aggregation of p25alpha. J Mol Biol 421:601-615. 10.1016/j.jmb.2012.01.050

Nilsson MR. 2004. Techniques to study amyloid fibril formation in vitro. Methods 34:151-160. 10.1016/j.ymeth.2004.03.012

O'Brien EP, Christodoulou J, Vendruscolo M, Dobson CM. 2011. New scenarios of protein folding can occur on the ribosome. J Am Chem Soc 133:513-526. 10.1021/ja107863z

O'Brien EP, Hsu ST, Christodoulou J, Vendruscolo M, Dobson CM. 2010. Transient tertiary structure formation within the ribosome exit port. J Am Chem Soc 132:16928-16937. 10.1021/ja106530y

O'Brien RJ, Wong PC. 2011. Amyloid precursor protein processing and Alzheimer's disease. Annu Rev Neurosci 34:185-204. 10.1146/annurev-neuro-061010-113613

O'Nuallain B, Wetzel R. 2002. Conformational Abs recognizing a generic amyloid fibril epitope. Proc Natl Acad Sci U S A 99:1485-1490. 10.1073/pnas.022662599

Powers ET, Powers DL. 2008. Mechanisms of protein fibril formation: nucleated polymerization with competing off-pathway aggregation. Biophys $J$ 94:379-391. 10.1529/biophysj.107.117168

Ramshini H, Parrini C, Relini A, Zampagni M, Mannini B, Pesce A, Saboury AA, Nemat-Gorgani M, Chiti F. 2011. Large proteins have a great tendency to aggregate but a low propensity to form amyloid fibrils. PLoS One 6:e16075. 10.1371/journal.pone.0016075

Richardson JS, Richardson DC. 2002. Natural beta-sheet proteins use negative design to avoid edge-toedge aggregation. Proc Natl Acad Sci U S A 99:2754-2759. 10.1073/pnas.052706099

Saab-Rincon G, Juarez VR, Osuna J, Sanchez F, Soberon X. 2001. Different strategies to recover the activity of monomeric triosephosphate isomerase by directed evolution. Protein Eng 14:149-155.

Scarafone N, Pain C, Fratamico A, Gaspard G, Yilmaz N, Filee P, Galleni M, Matagne A, Dumoulin M. 2012. Amyloid-like fibril formation by polyQ proteins: a critical balance between the polyQ length and the constraints imposed by the host protein. PLoS One 7:e31253. 10.1371/journal.pone.0031253 
564

565

566

567

568

569

570

571

572

573

574

575

576

577

578

579

580

581

582

583

584

585

586

587

588

589

590

591

592

593

594

595

596

597

598

599

600

601

602

603

604

605

606

607

608

609

610

Schliebs W, Thanki N, Jaenicke R, Wierenga RK. 1997. A double mutation at the tip of the dimer interface loop of triosephosphate isomerase generates active monomers with reduced stability. Biochemistry 36:9655-9662.

Serio TR, Cashikar AG, Kowal AS, Sawicki GJ, Moslehi JJ, Serpell L, Arnsdorf MF, Lindquist SL. 2000. Nucleated conformational conversion and the replication of conformational information by a prion determinant. Science 289:1317-1321.

Shivu B, Seshadri S, Li J, Oberg KA, Uversky VN, Fink AL. 2013. Distinct beta-sheet structure in protein aggregates determined by ATR-FTIR spectroscopy. Biochemistry 52:5176-5183. $10.1021 / \mathrm{bi} 400625 \mathrm{v}$

Solomon JP, Yonemoto IT, Murray AN, Price JL, Powers ET, Balch WE, Kelly JW. 2009. The 8 and 5 kDa Fragments of Plasma Gelsolin Form Amyloid Fibrils by a Nucleated Polymerization Mechanism, while the $68 \mathrm{kDa}$ Fragment is Not Amyloidogenic. Biochemistry 48:11370-11380. 10.1021/bi901368e

Tsolis AC, Papandreou NC, Iconomidou VA, Hamodrakas SJ. 2013. A consensus method for the prediction of 'aggregation-prone' peptides in globular proteins. PLoS One 8:e54175. 10.1371/journal.pone.0054175

Tzotzos S, Doig AJ. 2010. Amyloidogenic sequences in native protein structures. Protein Sci 19:327-348. $10.1002 /$ pro. 314

Ugrinov KG, Clark PL. 2010. Cotranslational folding increases GFP folding yield. Biophys $J$ 98:13121320. 10.1016/j.bpj.2009.12.4291

Vetri V, Canale C, Relini A, Librizzi F, Militello V, Gliozzi A, Leone M. 2007. Amyloid fibrils formation and amorphous aggregation in concanavalin A. Biophys Chem 125:184-190. 10.1016/j.bpc.2006.07.012

Walsh I, Seno F, Tosatto SC, Trovato A. 2014. PASTA 2.0: an improved server for protein aggregation prediction. Nucleic Acids Res. 10.1093/nar/gku399

Wang L, Schubert D, Sawaya MR, Eisenberg D, Riek R. 2010. Multidimensional structure-activity relationship of a protein in its aggregated states. Angew Chem Int Ed Engl 49:3904-3908. 10.1002/anie.201000068

Westermark P. 2005. Aspects on human amyloid forms and their fibril polypeptides. FEBS J 272:59425949. 10.1111/j.1742-4658.2005.05024.x

Wierenga RK. 2001. The TIM-barrel fold: a versatile framework for efficient enzymes. FEBS Letters 492:193-198. http://dx.doi.org/10.1016/S0014-5793(01)02236-0

Wu C, Shea JE. 2011. Coarse-grained models for protein aggregation. Curr Opin Struct Biol 21:209-220. 10.1016/j.sbi.2011.02.002

Zandomeneghi G, Krebs MRH, McCammon MG, Fändrich M. 2004. FTIR reveals structural differences between native $\beta$-sheet proteins and amyloid fibrils. Protein Science : A Publication of the Protein Society 13:3314-3321. 10.1110/ps.041024904

Zerovnik E, Stoka V, Mirtic A, Guncar G, Grdadolnik J, Staniforth RA, Turk D, Turk V. 2011. Mechanisms of amyloid fibril formation--focus on domain-swapping. FEBS J 278:2263-2282. 10.1111/j.1742-4658.2011.08149.x

Zhang X, Dong Y, Yu J, Tu X. 2014. Effects of environmental factors on MSP21-25 aggregation indicate the roles of hydrophobic and electrostatic interactions in the aggregation process. Eur Biophys $J$ 43:1-9. 10.1007/s00249-013-0934-9

Zou Y, Hao W, Li H, Gao Y, Sun Y, Ma G. 2014. New insight into amyloid fibril formation of hen egg white lysozyme using a two-step temperature-dependent FTIR approach. J Phys Chem B 118:9834-9843. 10.1021/jp504201k

\section{Figure Legends}


611 Figure 1. Aggregation kinetics followed by A) ThT fluorescence and B) by turbidimetry at 405

$612 \mathrm{~nm}$ of HsTPIn (dashed line) and HsTPIurea (solid line). C) TEM images of HsTPI aggregates at

613 the final time point of aggregation. Scale bars are $1 \mu \mathrm{M}$.

614 Figure 2. Secondary structure of HsTPI aggregates. A) Second-derivative ATR-FTIR spectra in

615 the amide I region of salted-out HsTPI (dotted line), HsTPIn (dashed line) and HsTPIurea (solid

616 line). B) Dot-blot assay of HsTPI aggregates with the WO1 antibody confirming cross- $\beta$

617 structure.

618

619

620

621

622

623

624

625

626

627

628

629

630

631

Figure 3. Identification of potential fibrillogenic regions in HsTPI. A) Consensus of different prediction methods of fibrillogenic regions. The $\beta$-strands are shown in green, the $\alpha$-helices in yellow and the chameleonic sequences are underlined. Aspartic residues are in red, indicating the potential sites for acid hydrolysis. Prediction hits are shown in the corresponding line of the predictor. B) List of the potential fibrillogenic regions selected for peptide aggregation assay. All $\beta$-strands were selected as well as the three more significant chameleonic sequences of the protein.

Figure 4. Peptide aggregation. A) ThT fluorescence intensities at $485 \mathrm{~nm}$ of aggregates of peptides at final time point of incubation. B) Congo red birefringence assay of the $\beta 3$ (solid black line), $\beta 6$ (dashed line), $\beta 7$ (dotted-dashed line) and CS-3 (dotted line) aggregates. A maximal peak at $540 \mathrm{~nm}$ is shown in aggregates compared with Congo red alone (solid gray line).

Figure 5. TEM images of peptides aggregates. The scale bar are $1 \mu \mathrm{m}$.

Figure 6. Acid hydrolysis of HsTPI. A) Tricine SDS-PAGE of hydrolyzed HsTPI. B) Aggregation kinetics of the hydrolyzed fragments of HsTPI followed by ThT fluorescence. C) 
632 TEM image of the amyloid fibrils formed by fragmented HsTPI; the scale bars is $1 \mu \mathrm{M}$. D)

633 Tricine SDS-PAGE of the enriched fragment upon aggregation. E) MS/MS spectrum of the triply

634 charged precursor ion at m/z 1048.84 identifies the amyloid fragment as the sequence

635 DPKIAVAAENCYKVTNGAFTGEISPGMIKD, which corresponds to residues 57-85 of HsTPI.

636 
637 Figure 1

A)

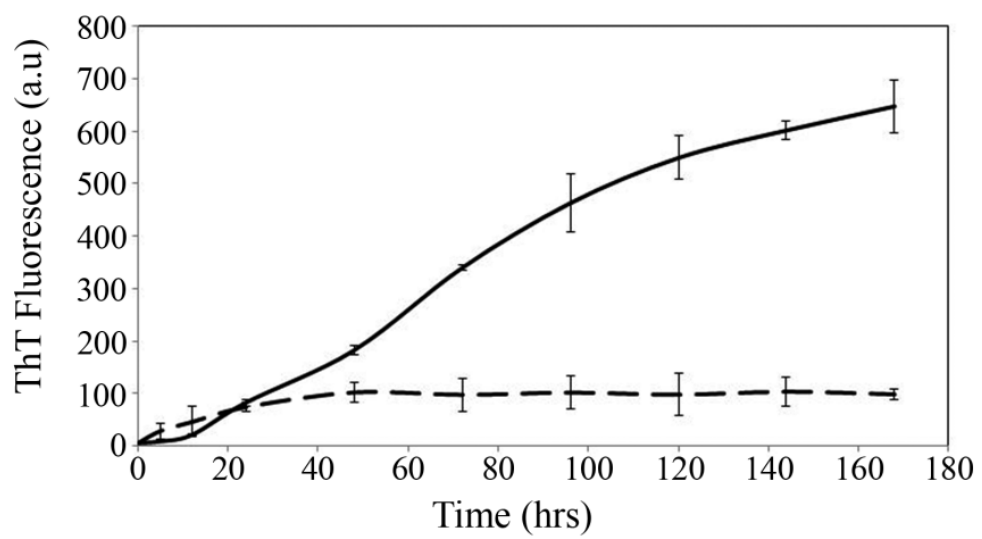

B)

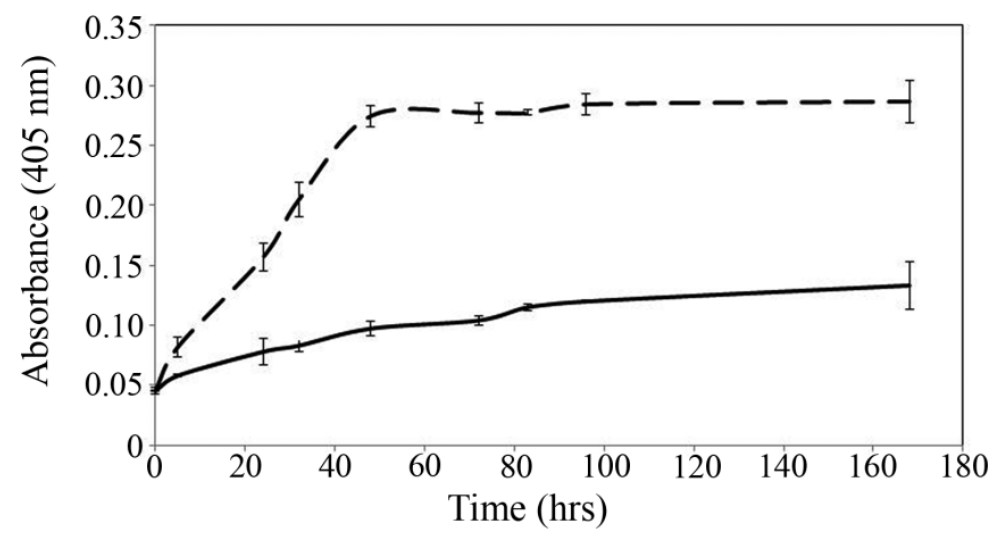

C)

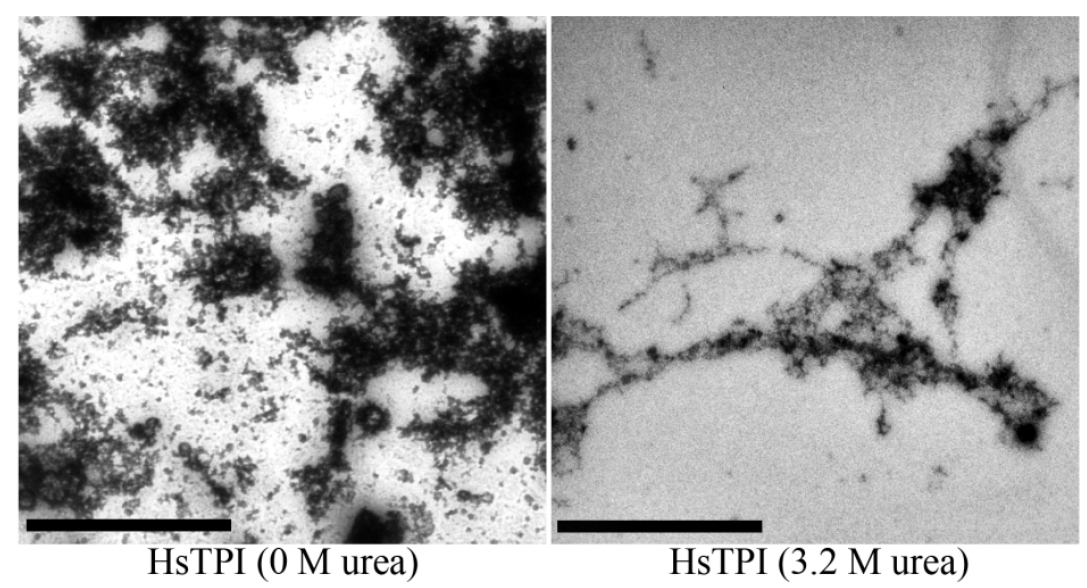


640 Figure 2

641

A)

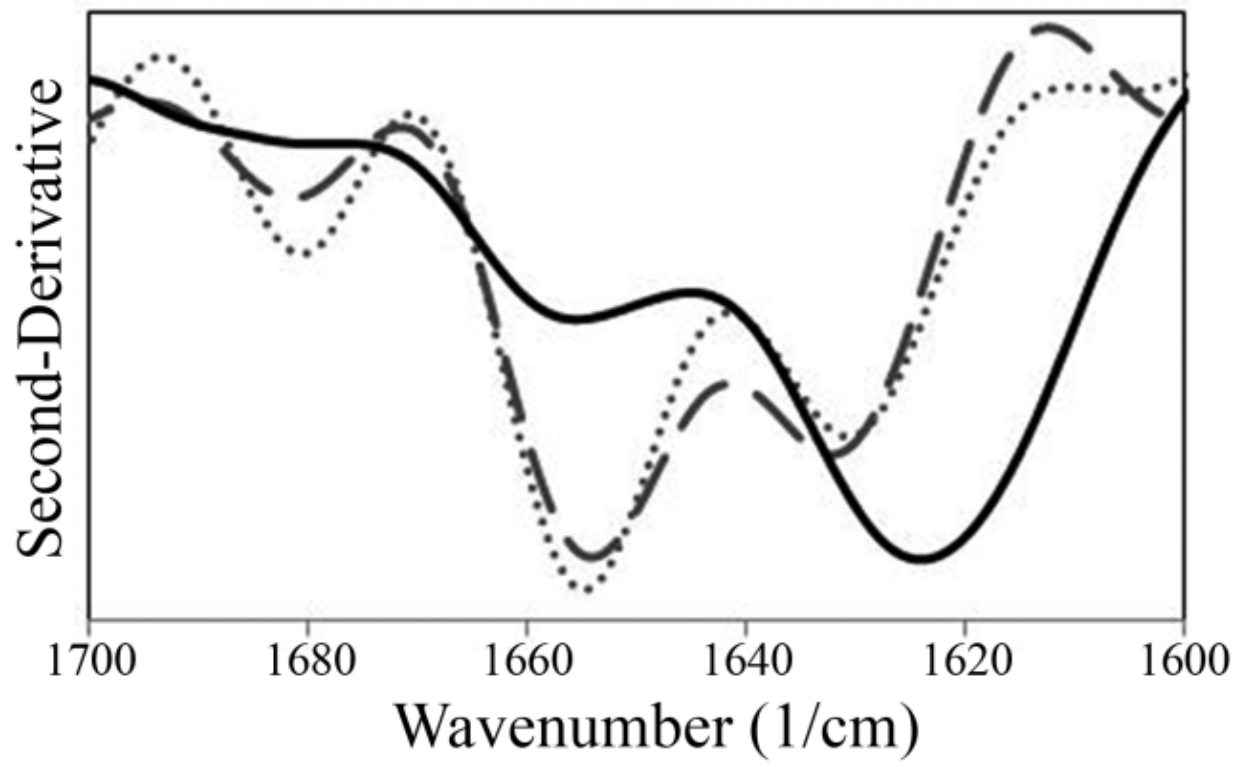

B)

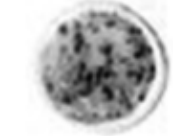

HsTPI aggregates

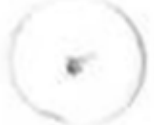

Soluble HsTPI

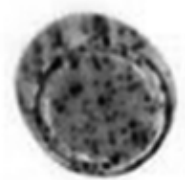

Lysozyme aggregates

642 


\section{Figure 3}

A)

FISH AMYLOID

FOLD AMYLOID

AMYLPRED 2

PASTA 2.0

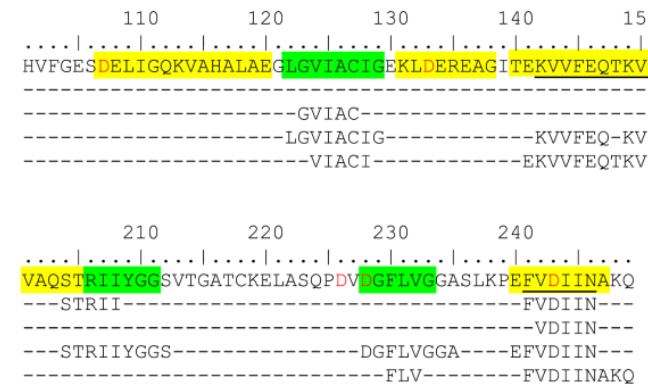

$50 \quad 60$

60

70

80

$80 \quad 90 \quad 100$ MAPSRKFFVGGNWKMNGRKQSLGELIGTLNAAKVPA TEVVCAPPTAYIDFARQKLDPKIAVAAQNCYKVTNGAFTGEISPGMIKDCGATWVVLGHSERR

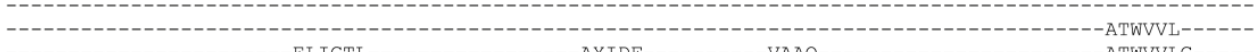

$\begin{array}{lllll}150 & 160 & 170 & 180 & 190\end{array}$ .|... $|\ldots| \ldots|\ldots| \ldots|\ldots| \ldots|\ldots| \ldots \mid$

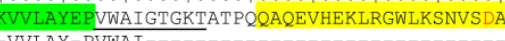
-VVLA--PVWAI-

FISH FOLD AMYLOI

AMYLPRED 2

PASTA 2.0

FISH AMYLOID
FOLD AMYLOID
AMYLPRED 2

AMYLPRED 2
B)

$\begin{array}{ll}1 . \beta 1 & (6-14) \\ 2 \cdot \beta 2 & (37-45) \\ 3 \cdot \beta 3 & (59-66) \\ 4 \cdot \beta 4 & (90-96) \\ 5 \cdot \beta 5 & (120-130) \\ 6 . \beta 6 & (160-167)\end{array}$

7. $\beta 7 \quad(203-214)$

8. $\beta 8 \quad(226-236)$

9. $C S-1 \quad(141-153)$

10. CS-2 (168-176)

11. CS-3 (239-249)

645

646 
647 Figure 4

A)

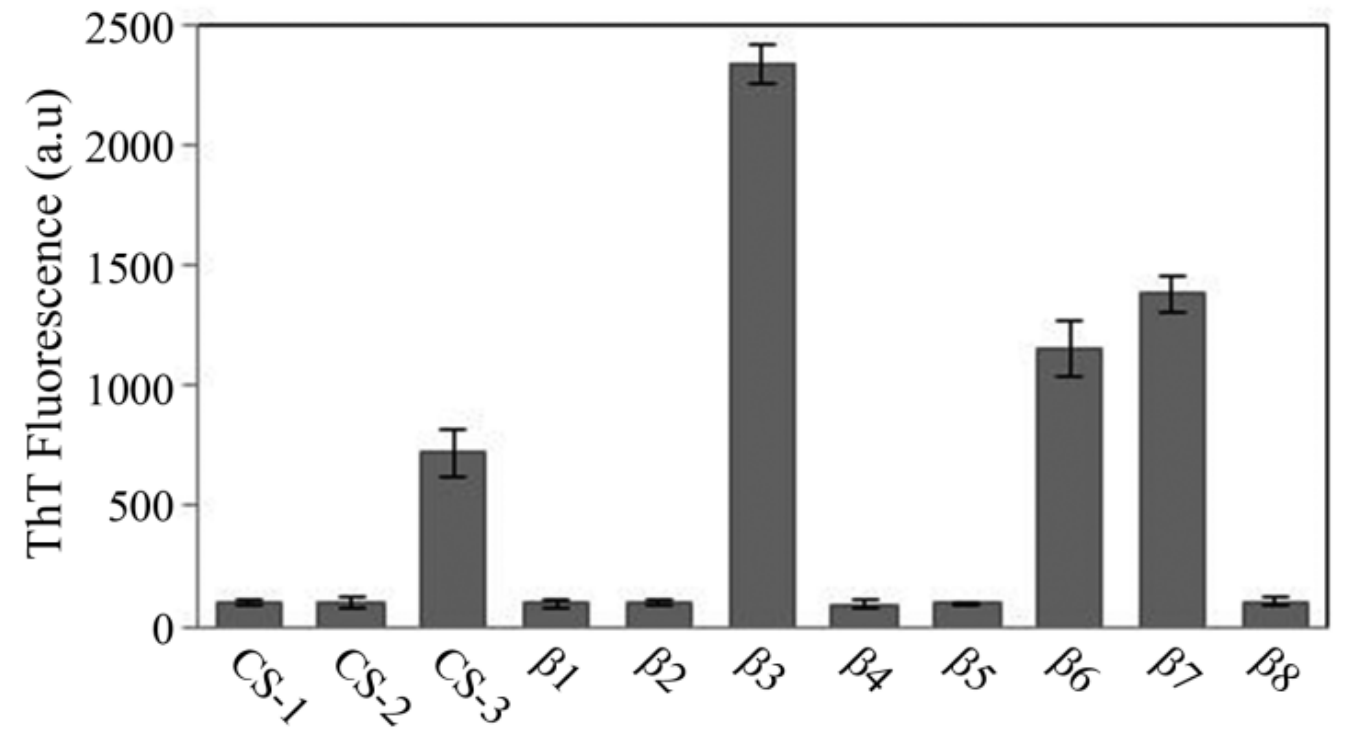

B)

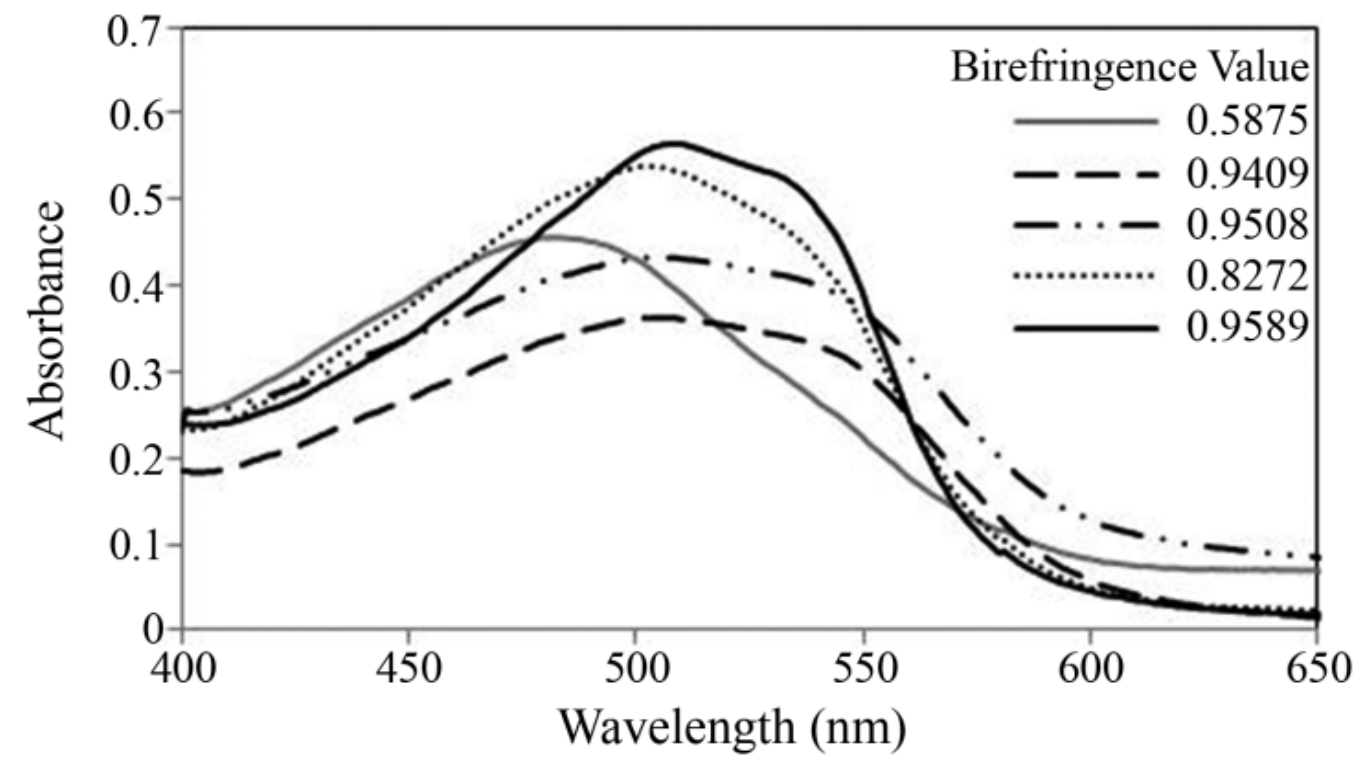


650 Figure 5

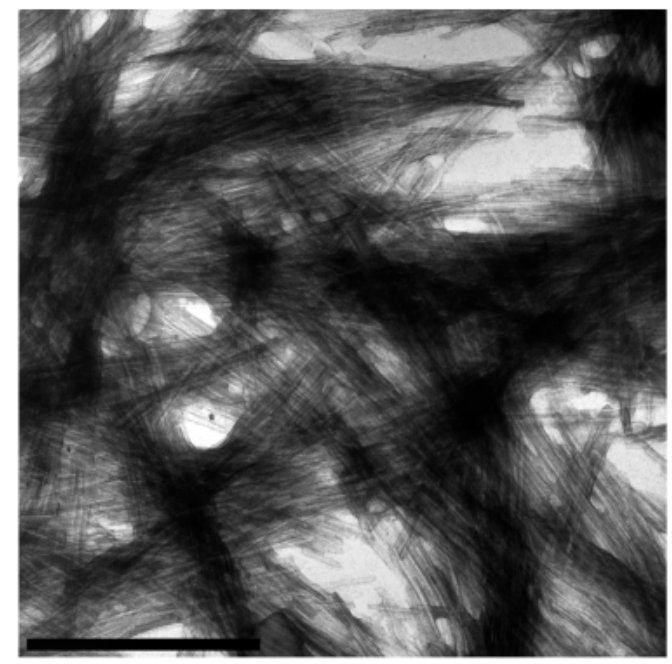

$\beta 3(59-66)$

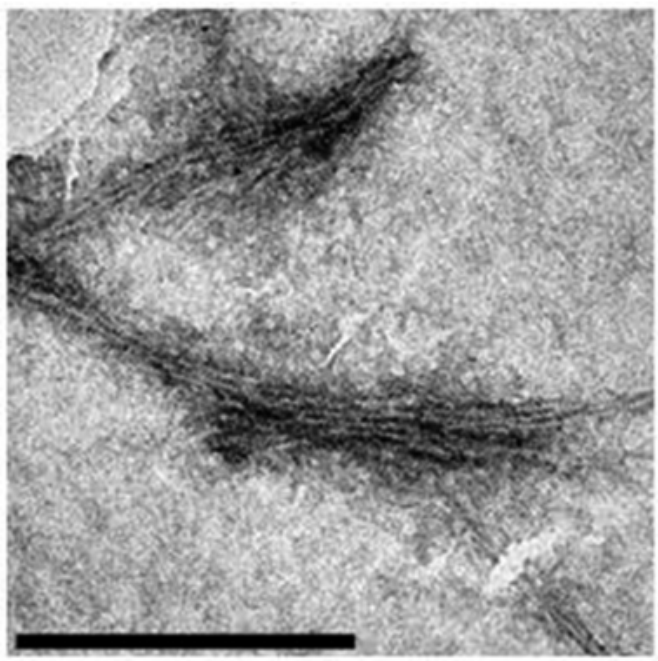

$\beta 7(203-214)$

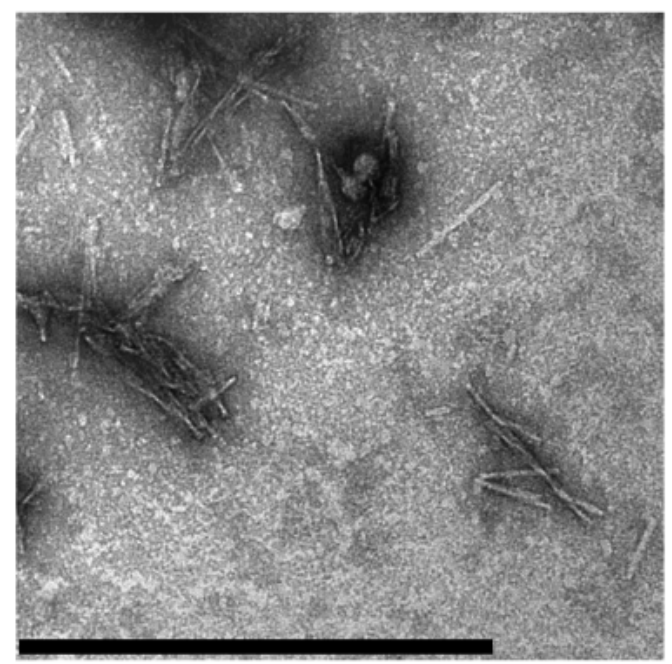

$\beta 6(160-167)$

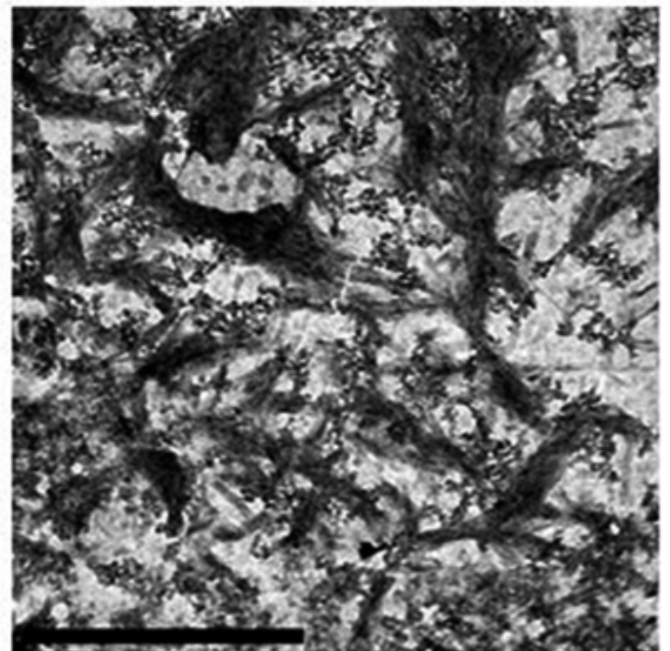

CS-3(239-249)

651

652 
653 Figure 6

A)

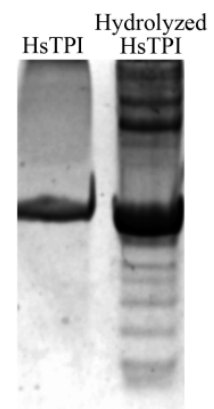

D)

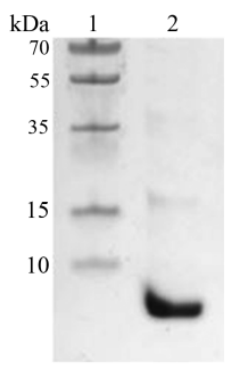

B)

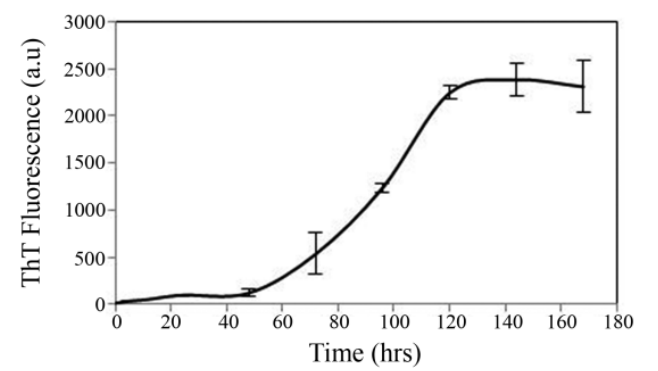

E)

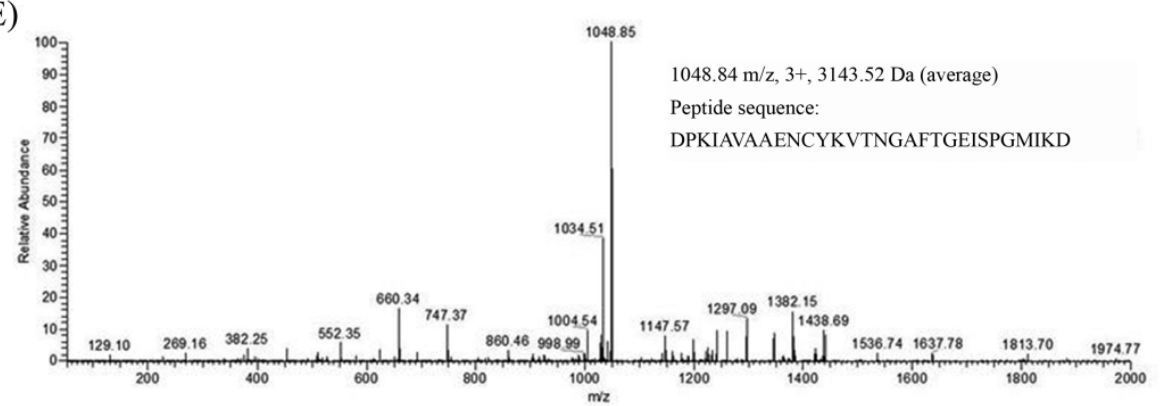

C)

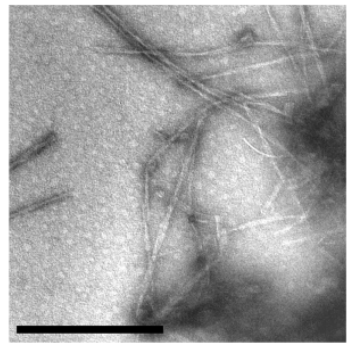

eptide sequence: 
656 Table S1

\begin{tabular}{|c|c|c|c|c|c|}
\hline No & Name & Sequence & Region & Length (residues) & charge \\
\hline 1 & CS-1 & EKVVFEQTKVIAD & $141-153$ & 13 & -1 \\
\hline 2 & CS-2 & VWAIGTGKT & $168-176$ & 9 & 1 \\
\hline 3 & CS-3 & PEFVDIINAKQ & $239-249$ & 11 & -1 \\
\hline 4 & $\beta 1$ & KFFVGGNWK & $6-14$ & 9 & 2 \\
\hline 5 & $\beta 2$ & DTEVVCAPP & $37-45$ & 9 & -2 \\
\hline 6 & $\beta 3$ & KIAVAAQN & $59-66$ & 8 & 1 \\
\hline 7 & $\beta 4$ & TWVVLGH & $90-96$ & 7 & 1 \\
\hline 8 & $\beta 5$ & EGLGVIACIGE & $120-130$ & 11 & -2 \\
\hline 19 & $\beta 6$ & KVVLAYEP & $160-167$ & 8 & 0 \\
\hline 10 & $\beta 7$ & QSTRIIYGGSVT & $203-214$ & 12 & 1 \\
\hline 11 & $\beta 8$ & DVDGFLVGGAS & $226-236$ & 11 & -2 \\
\hline
\end{tabular}

658 Table S1. Synthetic peptides tested in aggregation assays.

659 
660 Figure S2

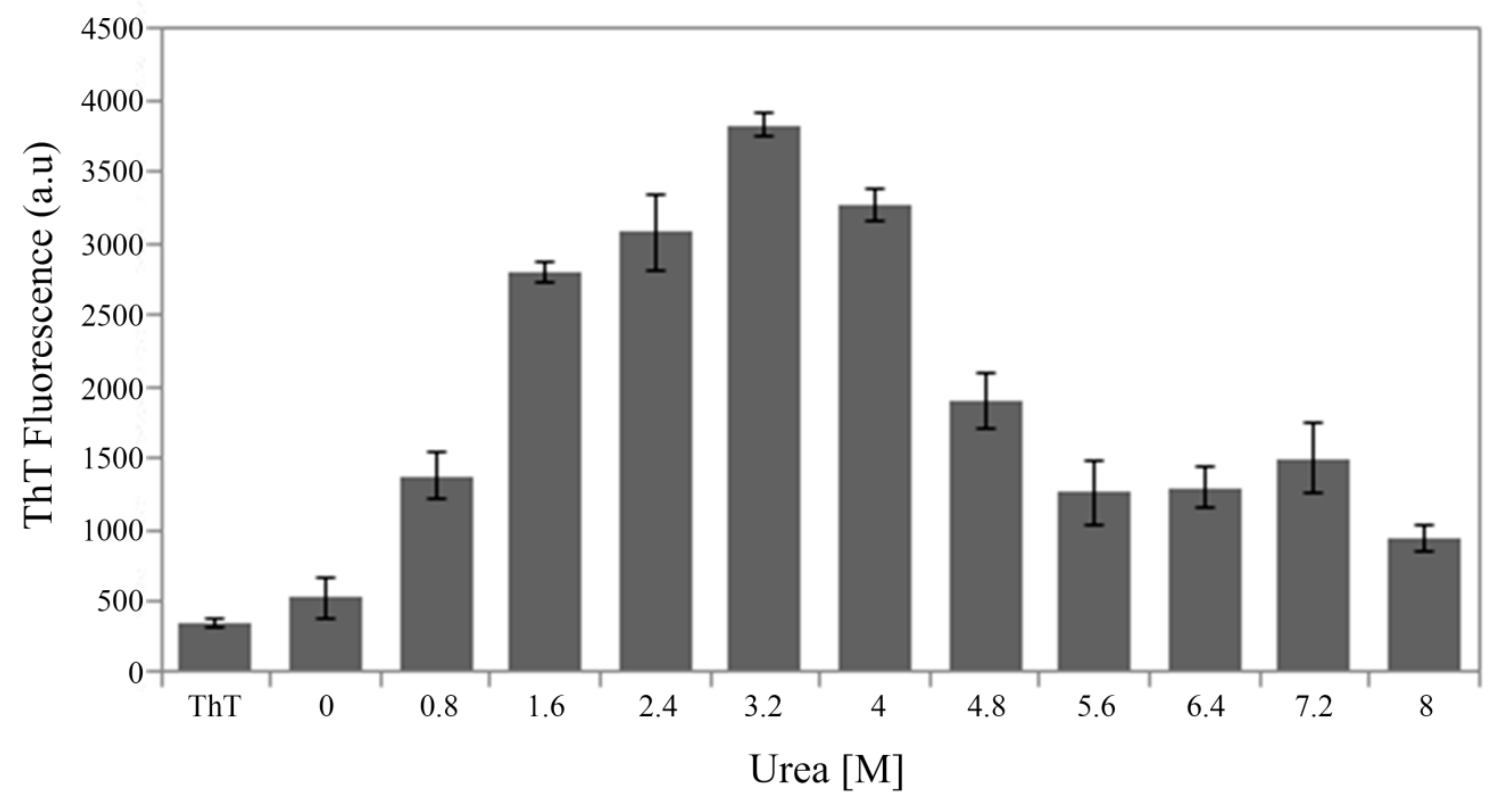

661 